\title{
ANNA SZEMBERSKA
}

Università Adam Mickiewicz, Poznań

annas@amu.edu.pl

\section{COMPOSTI NOME-NOME COORDINATI E SUBORDINATI NEL LESSICO SPORTIVO ITALIANO COME PROVA DELLE TENDENZE IN ATTO NELL'ITALIANO CONTEMPORANEO}

\begin{abstract}
Anna Szemberska, I composti Nome-Nome coordinati e subordinati nel lessico sportivo italiano come prova delle tendenze in atto nell'italiano contemporaneo [Noun-Noun Compounds in Italian Sports Column as a Proof of Tendencies in Modern Italian], Studia Romanica Posnaniensia, Adam Mickiewicz University Press, Poznań, vol. XL/3: 2013, pp. 95-103. ISBN 978-83-232-2638-3. ISSN 01372475. eISSN 2084-4158. DOI: 10.7169/strop2013.403.008

The compounding is a fundamental process of word formation and its importance to our understanding of language is crucial. The study of compounds for the last few years has been at the centre of attention in such areas of linguistics as computational approaches, psycholinguistics and language acquisition. Linguists advanced hypotheses not only regarding the construction of compounds, but also where they fit into the model of grammar. The paper attempts to answer some basic questions pertaining to lexical creativity in nominal compounding in Italian sports column. These include the definition of the concept, the types of noun-noun compounds in Modern Italian and a brief comparison of these types.
\end{abstract}

Keywords: Noun-Noun compounds, Italian sports column, Italian football lexicon, lexical creativity, headedness

\section{INTRODUZIONE}

In questa sede si intendono analizzare i composti seguenti lo schema NomeNome nell'italiano contemporaneo. A titolo d'introduzione occorre precisare che per la nozione di composti 'Nome-Nome' ci si allaccia a Liverani Bertinelli (1994). Notiamo pertanto che tali strutture vengono anche dette lessemi complessi (Voghera, 1994), mentre a parlare di unità lessicali superiori è Dardano (1978 nonché 1996). Pronińska (2005) ricorre alla nozione di polirematiche sostantivali e Pittano (1987) intanto parla in maniera più generale di parole neonuove, includendoci anche i composti Nome-Nome.

Visto l'argomento della trattazione, si è deciso di restringere l'analisi ai soli articoli presi dalla seguente testata elettronica': la meneghina La Gazzetta dello Sport

\footnotetext{
${ }^{1}$ Per il termine giornale elettronico ci si allaccia a Gualdo (2007: 121). I quotidiani on line sono la versione, più o meno completa, del quotidiano pubblicata su Internet (cfr. Sensini, 2005: 345).
} 
(gazzetta.it) di cui si sono analizzate le rubriche dedicate alla cronaca delle partite di calcio delle squadre italiane partecipanti al torneo della Champions League nel periodo tra il gennaio del $1997^{2}$ ed il dicembre del 2010 (il mese di dicembre del 2010 coincide con l'inizio dell'elaborazione della parte analitica del presente contributo) distribuendo le annate nella fase a gironi (che comprende il periodo da settembre a dicembre) e nella fase finale (da metà febbraio a maggio). Data l'impossibilità di trattare il considerevole numero di articoli scritti nel periodo tra il 1997 e la fine del 2010, si è deciso di suddividere ulteriormente il suddetto periodo della ricerca in sottoperiodi di due anni. Quel periodo ci pare rappresentativo. E così si sono anlizzati i seguenti periodi: autunno 2010 - primavera 2009, autunno 2006 - primavera 2005, autunno 2002 - primavera 2001 e autunno 1998 - primavera 1997.

La scelta della materia d'indagine perciò, nonché della restrizione del campo di ricerca ai soli composti nominali italiani del tipo Nome-Nome viene parzialmente dal seguente presupposto - puntualizzazione di Beszterda (2004: 110) che «la categoria dei nomi composti costituisce la maggior parte delle parole composte». Come risulta dalle rilevazioni soprastanti, il lessico nominale è una parte significativa e cospicua del lessico italiano. Tuttavia per via delle ragioni di spazio si è deciso di non approfondire la questione della diversità di vedute in merito alla delimitazione degli stessi composti nominali italiani ${ }^{3}$.

\section{I MODELLI INGLESI}

Volendo analizzare i composti Nome-Nome va tenuto presente che la classificazione dei composti avviene in base alla loro struttura morfosintattica (Beszterda, 2004: 110). Non irrilevante alle strutture che concernerà la nostra ricerca risulta essere l'influsso dei sintagmi angloamericani. Pertanto vale la pena osservare che sono numerosi gli studiosi [Severgnini (1997: 15 e 2007); Frenguelli (2005: 159-177); Giovanardi, Gualdo (2003) nonché Faustini (2006: 171)] a sottolineare che negli ultimi anni possiamo notare il crescente influsso delle strutture inglesi sul sistema linguistico italiano. Difatti, vista e considerata la relativamente facile riproducibilità dei sintagmi angloamericani del tipo collegamento Internet, indirizzo Internet, e, per attingere al linguaggio meramente giornalistico, governo Berlusconi, i neologismi rifacentisi alle strutture apparentemente non oriunde sembrano costituire una parte cospicua dell'italiano contemporaneo [Dardano, Frenguelli, Perna (2000: 31-55); nonché Dardano, Frenguelli, Puoti (2005: 229-248) ed anche Giovanardi, Gualdo (2003)]. I linguisti (Frenguelli, 2005: 164) in effetti rilevano che il suddetto settore della formazione

\footnotetext{
${ }^{2}$ Questa scelta è dettata dal fatto che l'archivio storico della versione telematica de La Gazzetta dello Sport risale a quell'anno.

${ }^{3}$ L'argomento viene trattato in Szemberska, "La composizione nominale in italiano: un'odierna Questione della lingua?" (forthcoming).
} 
delle parole dell'italiano contemporaneo, oggi notevolmente in espansione, si sarebbe modellato appoggiandosi alle strutture angloamericane, subendone una certa influenza. Tuttavia, come accennavamo sopra, una cospicua parte dei costrutti in questione, risultanti dallo spoglio della versione telematica del quotidiano italiano La Gazzetta dello Sport appartiene ai composti ibridi angloitaliani, perciò ed anche per via del fatto che su di essi si sono modellati e/o vanno modellandosi mediante la trasposizione di modelli sintattici ${ }^{4}$, come si menzionava inizialmente, i composti Nome-Nome ad ambedue gli elementi italiani. Notiamo intanto che i soprammenzionati composti ibridi angloitaliani, ovvero formati da un elemento italiano e da uno inglese, come prontamente non manca di notare Frenguelli (2005: 159), risulterebbero piuttosto assenti nei lavori dei linguisti italiani e degli italianisti in genere.

Tornando al concetto di prima, a titolo d'esempio di composti italiani modellatisi sull'inglese possiamo citare due composti nominali contenenti il lessema ragazza: call girl/ragazza squillo (Pronińska, 2005: 70), cover girl/ragazza copertina. Come risulta dagli esempi, le due forme di frequente coesistono per poi lasciar spazio a quella dominante, la quale, dopo qualche tempo, risulta spesso essere nientemeno che il costrutto italianizzato (Pronińska, 2005: 70-71). Ciononostante va osservato che i composti di questo tipo, pur alquanto diffusi nel linguaggio dei mass media e, nella fattispecie, in quello dei giornali, raramente diventano occorrenze nei vocabolari. In effetti, il riserbo e la non sempre pronta disponibilità ad accoglierli come nuove entrate sarebbero dovuti alla loro vera o presunta provvisorietà nonché alla non conformità o persino lontananza dalla norma scolastica. Un certo peso pare avere anche la loro formazione relativamente recente e non di rado specialistica (Frenguelli, 2005: 160). Tuttavia, è un dato di fatto che mentre negli ultimi anni i composti con elementi neoclassici sono rimasti un insieme piuttosto costante, almeno nella lingua comune, i composti ibridi angloitaliani appaiono in espansione (Frenguelli, 2005: 163-164).

Un altro efficace esempio della rilevanza del fenomeno è costituito dalle recenti ricerche di Adamo, Della Valle (2005: X), dai dati dei quali emergono 495 parole nuove provenienti dai quotidiani italiani. Effettivamente gli autori stessi affermano (2005: VII) che le parole nuove da loro raccolte risultano dalla

lettura sistematica di trentanove quotidiani, da quelli di maggior diffusione a quelli che documentano le principali realtà regionali italiane o le più rappresentative tendenze politiche e sociali, con la preoccupazione di testimoniare l'arricchimento del mosaico variegato della lingua nazionale, per un totale di 3.623 brani firmati da 1.084 giornalisti.

Nonostante non siamo in grado di dare risposte concrete per quanto si tratti di parole stabili o di creazioni effimere non destinate a durare, bisogna considerare la loro rilevanza tipologica.

4 Sulla nozione di trasposizione di modelli sintattici nonché sull'argomento di adattamenti semantici e calchi fraseologici cfr. Pronińska (2005: 70) ed anche Frenguelli (2005). 
Da notare anche che a parere di alcuni linguisti (Frenguelli, 2005: 160) la tendenza di cui trattiamo in questa sede avrebbe rispondenza in altre lingue dell'Europa Occidentale, quali il francese ed il tedesco. Osserviamo inoltre che la composizione ibrida avrebbe contribuito a sviluppare nonché a propagare nell'italiano contemporaneo la successione determinante + determinato. Paragoniamo i seguenti costrutti riportati dallo stesso Frenguelli (2005: 164):

baby consulenti - «consulenti molto giovani», kick aerobica - «aerobica eseguita con movimenti di kickboxing»

con

pensione baby - «pensione giovanile, attribuita a persona molto giovane» o batterio killer «batterio che uccide».

Notiamo (Frenguelli, 2005: 164-165) che la suddetta successione di parole differisce da quella dominante nel sistema linguistico italiano vent'anni orsono quando l'ordine romanzo era per lo più rispettato: volo charter, industria leader, musica jazz. I modelli inglesi dei composti sopraelencati sarebbero rispettivamente: charter flight, leader industry e jazz music. Con il passare degli anni la successione determinante + determinato, ovverosia, come abbiamo già sottolineato, l'ordine non romanzo, ha conquistato nuovi spazi.

\section{IL SIGNIFICATO UNITARIO}

I linguisti non di rado sottolineano che il criterio dell'essere composto è il significato unitario dei costituenti del costrutto. Notiamo che in merito ai composti in generale Hartmann, James (1998: 97) mettono in rilievo l'importanza della compattezza semantica dei formanti: «The constituents are relatively stable (fixed expression), and, if used idiomatically, their combined meaning is more or other than the sum of the parts, eg. fly-by-night, face the music». La compattezza semantica sembra essere compatibile con il concetto di significato unitario (Gusmani, 1979; Pronińska, 2005).

Come si può osservare, il concetto del significato unitario coinciderebbe grossomodo con la concezione di unità semantica dei costituenti di un composto, riscontrabile in Voghera (1994), mentre in Tollemache (1945) ci si troverà l'unità dell'immagine.

\section{I COMPOSTI NOME-NOME}

\subsection{COMPOSTI NOME-NOME COORDINATI}

Il rapporto che intercorre tra gli elementi formanti è di coordinazione: portieri protagonisti: «E il resto è puro contorno coi due portieri protagonisti che negano altrettanti gol a Zamorano e Haas» (10/12/1998) [1], paese organizzatore: «Ma le 
fasi finali degli Europei e dei Mondiali generano grandissimi ricavi per Paese organizzatore» (06/04/2006) [2], squadra campione: «Un posto nella squadra campione d'Italia val bene un passo indietro, una chiusura in più e un lancio d'autore in meno» (07/03/2001) [3], trequartista maestro: «Il quadrilatero a forma di rombo ha funzionato al meglio: Baumann si è confermato playmaker compassato ma sapiente; Micoud è stato trequartista maestro nel mimetizzarsi e nell'apparire dentro il metro quadro libero; Frings e Borowski hanno coperto al meglio i lati del castello» (03/11/2005) [4], palle-sponde: "Ancora straordinaria la sua prova. Ha messo praticamente il becco in quasi tutte le azioni di attacco della Juve, ha segnato un gol, ha dato palle - sponde importanti ai compagni e soprattutto ha messo sempre a soqquadro la difesa dell'Ajax» (10/04/1997) [5]. Puntualizziamo che i composti nominali di questo tipo vengono anche definiti composti copulativi in quanto in essi la relazione tra gli elementi costitutivi potrebbe essere esplicitata da un verbo copula (Beszterda, Sypnicki, 2004: 43). Morfologicamente questo tipo di composti viene formato dal congiungimento di due sostantivi ed i loro membri sono equipotenti in quanto la funzione di determinante pare essere reciproca.

Bisognerebbe anche far notare un'altra possibile sfumatura di significato all'interno dei composti Nome-Nome coordinati, ovvero quando le relazioni intercorrenti tra $\mathrm{i}$ formanti si potrebbero esplicitare tramite due frasi legittime in quanto $\mathrm{N}_{1}$ è $\mathrm{N}_{2}$, e, allo stesso tempo, $\mathrm{N}_{2}$ è $\mathrm{N}_{1}$ (Beszterda, Sypnicki, 2004: 43-44), come nel caso del composto portieri protagonisti. Sul succitato esempio al contempo osserviamo gli stessi predicati (essere portieri ed essere protagonisti) implicare la differentia specifica che doveva costituire l'iponimo. Ne risulterebbe che la relazione di iper-/iponimia non concernerebbe i tipi esaminati. Difatti, Beszterda, Sypnicki (2004: 43-44) analizzando un analogo caso francese suggeriscono di presupporre l'assenza dell'iperonimo nel composto oppure di ipotizzare l'esistenza dei due iperonimi simultaneamente. Come esempio di una tale implicazione gli autori riportano il composto nominale francese acteur-auteur il quale corrisponderebbe rispettivamente a essere attore ed essere autore. Allo stesso tempo la suddetta ambiguità strutturale può essere eliminata in maniera pragmatica, supponendo che l'utente della lingua sia consapevole del fatto che l'individuo in questione sia soprattutto attore e secondariamente autore o viceversa.

\subsection{COMPOSTI NOME-NOME SUBORDINATI}

In questo tipo di composti si può distinguere il determinato o, conformemente alla linguistica generativista (Scalise, 1994: 133), l'elemento testa/la testa, e il determinante: inizio campionato: «In più c'è Vargas, l'extraterrestre di questo inizio campionato viola» (25/11/2009) [6], lezione Real: «Capello, altra lezione Real / La Roma cambia modulo per ovviare alle assenze, ma il tris dei campioni d'Europa la manda ancora k.o.» (18/09/2002) [7], linea-young: «Benitez azzarda la «linea young» 
e lancia Biabiany e Coutinho» (29/09/2010) [8], conferenza stampa: «Gli intoppi non accadono soltanto in campo o ai protagonisti, ma anche agli addetti ai lavori, a fine partita, prima della tradizionale conferenza stampa» (21/02/2001) [9], «Alla conferenza stampa ha evitato tutte le domande dei giornalisti russi» $(05 / 11 / 1998)$ [10]. I composti equivalgono rispettivamente a inizio del campionato, lezione impartita dal Real, linea degli young, conferenza per la stampa ${ }^{5}$. Questo tipo di composti nella maggioranza dei casi viene formato tramite l'elissi della preposizione. Nel corpus sono stati tra l'altro rilevati i seguenti composti Nome-Nome subordinati: poker Inter: «(titolo): Poker Inter col Werder» (29/09/2010) [11], possesso palla: «Marin ha qualità, ma mica può giocare da solo, specie se il possesso palla è quasi sempre interista» (29/09/2010) [12], tabellino marcatori: «I nomi nel tabellino marcatori sono i soliti, e dire che in formazione c'erano delle novità: Milito out da stamattina (quanto si sarà mangiato le mani a vedere la difesa del Werder?), Pandev in tribuna» (29/09/2010) [13] ed anche i composti con i nomi delle squadre di calcio: naufragio Juventus: «(titolo): Naufragio Juventus / E' atterrata subito da Tristan e non entra mai in partita, poi Davids si fa espellere ed è finita. Preoccupato di rinfrescare la squadra, Lippi la stravolge» (28/02/2002) [14], Milan batticuore: «(titolo): Milan batticuore / Il palo salva 1'1-1 con il Marsiglia» (26/11/2009) [15]. Frequenti sono anche i composti designanti le singole zone del campo: zona tiro: «Recoba, che pare il più pimpante del trio, dà la sensazione di poter spaccare il mondo ma poi tra lui e la zona tiro ci sono sempre troppi metri e troppi avversari perché le sue azioni possano rivelarsi pericolose» (03/10/2002) [16], metà campo: «Quattro a zero al Werder, di autorità, e il merito di Benitez è di aver sciolto ulteriormente la squadra, che ora gioca tenendo per sé il pallone e aggredendo la metà campo avversaria» (29/09/2010) [17].

\section{NOTE CONCLUSIVE}

Agli scopi posti nel presente articolo abbiamo analizzato le cronache delle partite di calcio italiane della versione telematica del quotidiano sportivo La Gazzetta dello Sport, ovvero gazzetta.it. Osserviamo che dal suddetto spoglio risulta che i composti Nome-Nome costituiscono un fenomeno rilevante e ben presente nell'italiano contemporaneo. Notiamo che i composti subordinati sono più numerosi di quelli coordinati: in effetti, mentre tra i primi contiamo 175 occorrenze nel corpus, $i$ composti coordinati sono appena 58. Si noti che i composti subordinati non in tutti i casi costituiscono un modo di comunicare efficace: qualche volta la loro interpretazione non risulta ovvia (milioni [da / della] Champions: «(titolo): MILIONI CHAMPIONS / Se il ritmo del primo tempo era stato blando, la ripresa sembrava proporre un Valencia più vivace, con Joaquin e Jorge Lopez (suo l'unico spunto che ha fatto correre un brivido a Doni:

\footnotetext{
5 Per ulteriori approfondimenti su questo tipo di composti si confronti Szemberska (2011:
} 6-7). 
16') abili in percussione» (06/12/2006) [18], fan club - club dei / per i fan: «Sono questi due a dare la scossa, a guidare la Lazio anche in una serata anonima d'autunno, dove fanno notizia lo striscione di un fan club polacco e la presenza in tribuna del sosia giovane di Zeman, che poi è «Sdengo», figlio della sorella del boemo-napoletano» (08/11/2000) [19]. Vediamo che la lingua tende sempre di più all'economia (l'omissione degli elementi di giunzione - preposizioni semplici nonché preposizioni articolate ed il risparmio di morfemi). Inoltre, i dati rilevati, ovvero la cospicua presenza dei composti Nome-Nome, corrispondono ai bisogni nominativi di una lingua settoriale. E' sorprendente che le strutture analizzate non riguardino solamente le azioni proprie del gioco di calcio: in molti casi si tratta del designare in maniera creativa un atteggiamento (Capello-pensiero: «Dall'altra parte, ci sarà Zinedine Zidane, che anche stavolta si è dimostrato un grandissimo campione: "Zizou era in una serata da Zizou - è il Capello-pensiero - con Roberto Carlos è stato l'uomo più importante per il Real. $\mathrm{Al}$ ritorno bisognerà stare molto attenti a entrambi. E soprattutto al contropiede, che è il pericolo numero uno"» (23/02/2005) [20].

\section{BIBLIOGRAFIA}

Adamo, Giovanni; Della Valle, Valeria (2005): 2006 parole nuove. Un dizionario di neologismi dai giornali, Milano: Sperling \& Kupfer Editori.

BESZTERDA, Ingeborga (2004): «Nomi composti in italiano e in francese: sistemi linguistici a confronto», Lingue e letterature in contatto. Associazione Internazionale Professori di Italiano, 3/2004, 1: 109-120.

BesZTERDA, Ingeborga; SYPNICKI, Józef (2004): «Alcune considerazioni inerenti alla natura dei composti in francese ed in italiano», in: M. Świątkowska, R. Sosnowski, I. Piechnik [eds.], Maestro e amico. Miscellanea in onore di Stanistaw Widlak, Kraków: Wydawnictwo Uniwersytetu Jagiellońskiego, 41-47.

Dardano, Maurizio (1978): La formazione delle parole nell 'italiano di oggi. (Primi materiali e proposte), Roma: Bulzoni Editore.

Dardano, Maurizio (1996): Manualetto di linguistica italiana, Bologna: Zanichelli.

Dardano, Maurizio; Frenguelli, Gianluca; Perna, Teresa (2000): «L'italiano di fronte all'inglese alle soglie del terzo millennio», in: S. Vanvolsem, D. Vermandere, Y. D’Hulst, F. Masara [eds.], L'italiano oltre frontiera. Atti del V Convegno internazionale (Leuven, 22-25/4/1998), 2 voll., vol. 1, Leuven-Firenze: Leuven University Press - Franco Cesati Editore, 31-55.

Dardano, Maurizio; Frenguelli, Gianluca; Puoti, Alberto (2005): «Anglofilia (para)testuale e morfologica?», in: I. Korzen, P. D'Achille [eds.], Tipologia linguistica e società. Due giornate italo-danesi di studi linguistici (Roma, 27-28/11/2003), Firenze: Franco Cesati Editore, 229-248.

Faustin, Gianni (2006): «Caratteristiche comuni», in: G. Faustini [ed.], Le tecniche del linguaggio giornalistico, Roma: La Nuova Italia Scientifica, 167-182.

Frengueldi, Gianluca (2005): «La composizione con elementi inglesi», in: C. Giovanardi [ed.], Lessico e formazione delle parole. Studi offerti a M. Dardano per il suo $70^{\circ}$ compleanno, Firenze: Franco Cesati Editore, 159-177.

GiovanARDI, Claudio; Gualdo, Riccardo (2003): Inglese-italiano 1 a 1. San Cesario di Lecce, Lecce: Manni Editori. 
Gualdo, Riccardo (2007): L'italiano dei giornali, Roma: Carocci.

GusmanI, Roberto (1979): «Calchi slavi», Incontri linguistici, 5: 147-156.

Hartmann, Reinhard Rudolf Karl; James, Gregory (1998): Dictionary of Lexicography, London New York: Routledge.

Liverani Bertinelli, Francesca (1994): L'italiano contemporaneo visto attraverso la stampa. Aspetti semantici del lessico e della morfosintassi, Perugia: Guerra Edizioni.

Pittano, Giuseppe (1987): «Passa-parola»-parole nuove e neonouve in economia e costume, Milano: Edizioni del Sole 24 ore.

PronińsKa, Aleksandra (2005): Principi teorici della compilazione del dizionario fraseologico italiano-polacco, Kraków: Wydawnictwo Naukowe AP.

SCALISE, Sergio (1994): Le strutture del linguaggio. Morfologia, Bologna: Il Mulino.

Sensin, Marcello (2005): La lingua e i testi. La riflessione sulla lingua, Milano: Arnoldo Mondadori Scuola.

Severgnini, Beppe (1997): «Ma anche gli inglesi scrivono "mafiosos" e "referendums"», Corriere della Sera, 16/05/1997: 15.

SevergninI, Beppe (2007): L'italiano. Lezioni semiserie, Roma: BUR Biblioteca Univ. Rizzoli.

SzEMBERSKA, Anna (2011): «Formazione delle parole nell'italiano contemporaneo sull'esempio del lessico calcistico». Romanica.doc, 3(4), URL: <http://www.romdoc.amu.edu.pl/Szemberska2. pdf $>$. Accessed on: 2011-09-30.

SzemBERSKA, Anna (forthcoming): «La composizione nominale in italiano: un'odierna Questione della lingua?». Atti del IV Convegno dei Giovani Italianisti "Sulle orme dell'unificazione italiana (lingua, letteratura, storia)", Toruń, Università M. Kopernik, 26-27/11/2011.

Tollemache, Federico (1945): Le parole composte nella lingua italiana, Roma: Edizioni Rores di Niccola Buffolo.

Voghera, Miriam (1994): «Lessemi complessi: percorsi di lessicalizzazione a confronto», Lingua e Stile, 29: 185-214.

URL:

http://archiviostorico.gazzetta.it/1998/dicembre/10/Inter_scalda_Graz_ga_0_9812106119.shtml. Accessed on: 2011-09-30.

http://archiviostorico.gazzetta.it/2006/aprile/06/Galliani_tengo_tutti_vita_ga_10_06040611294. shtml. Accessed on: 2011-09-30.

http://archiviostorico.gazzetta.it/2001/marzo/07/Lazio_cose_sul_serio_ga_0_0103077571.shtml. Accessed on: 2011-09-30.

http://archiviostorico.gazzetta.it/2005/novembre/03/Natale_illude_incredibile_Udinese_ga_10_ 0511032064.shtml. Accessed on: 2011-09-30.

http://archiviostorico.gazzetta.it/1997/aprile/10/una_Juve_troppo_forte_ga_0_9704102485.shtm1. Accessed on: 2011-09-30.

http://archiviostorico.gazzetta.it/2009/novembre/25/Delirio_viola_Fiorentina_agli_ottavi_ga_10 091125008.shtml. Accessed on: 2011-09-30.

http://archiviostorico.gazzetta.it/2002/settembre/18/Capello_altra_lezione_Real_ga_0_0209184913. shtml. Accessed on: 2011-09-30.

http://www.gazzetta.it/Calcio/SerieA/Inter/29-09-2010/triplo-eto-o-piu-sneijder-711297951919. shtml. Accessed on: 2011-09-30.

http://archiviostorico.gazzetta.it/2001/febbraio/21/Milan_salva_come_puo_autorete ga 0 0102216378.shtml. Accessed on: 2011-09-30.

http://archiviostorico.gazzetta.it/1998/novembre/05/come_avessimo_vinto_ga_0_9811052157.shtml. Accessed on: 2011-09-30. 
http://www.gazzetta.it/Calcio/SerieA/Inter/29-09-2010/triplo-eto-o-piu-sneijder-711297951919. shtml). Accessed on: 2011-09-30.

http://www.gazzetta.it/Calcio/SerieA/Inter/29-09-2010/triplo-eto-o-piu-sneijder-711297951919. shtml). Accessed on: 2011-09-30.

http://www.gazzetta.it/Calcio/SerieA/Inter/29-09-2010/triplo-eto-o-piu-sneijder-711297951919.shtml. Accessed on: 2011-09-30.

http://archiviostorico.gazzetta.it/2002/febbraio/28/Naufragio_Juventus_ga_0_0202288018.shtml. Accessed on: 2011-09-30.

http://archiviostorico.gazzetta.it/2009/novembre/26/Milan_batticuore_palo_salva_con_ga_10_ 091126103.shtml. Accessed on: 2011-09-30.

http://archiviostorico.gazzetta.it/2002/ottobre/03/Inter_scende_dal_treno_ga_0_0210032445.shtml. Accessed on: 2011-09-30.

http://www.gazzetta.it/Calcio/SerieA/Inter/29-09-2010/triplo-eto-o-piu-sneijder-711297951919.shtml. Accessed on: 2011-09-30.

http://archiviostorico.gazzetta.it/2006/dicembre/06/Panucci_timbra_pass_della_Roma_ga_10_ 061206077.shtml. Accessed on: 2011-09-30.

http://archiviostorico.gazzetta.it/2000/novembre/08/Lazio_con_minimo_sforzo_ga_0_00110814401. shtml. Accessed on: 2011-09-30.

http://archiviostorico.gazzetta.it/2005/febbraio/23/Possiamo_farcela_credo_ga_10_0502238973. shtml. Accessed on: 2011-09-30. 